\title{
Ionic Liquids in Chemical Analysis, CRC Press, 2009 a Compendium of Knowledge on ILs that Goes Well Beyond the Analytical Field
}

\author{
Grazyna Wilczek-Vera ${ }^{a}$ and Juan H. Vera ${ }^{\mathrm{b}, *}$
}

${ }^{a}$ Department of Chemistry, ${ }^{b}$ Department of Chemical Engineering, McGill University, Montreal, Que., Canada

\begin{abstract}
This note presents an overview of the development of literature in the field of ionic liquids (ILs) and related activities in this fast-growing field. In particular, we call the attention of those not working in the field of ILs to the text "Ionic Liquids in Chemical Analysis", CRC Press, 2009, Mihkel Koel, Editor, which contains important material of general interest. A brief survey of reviews published after the publication of this book is given.
\end{abstract}

Keywords: Ionic liquids, green chemistry, separation science, thermophysical properties, chemical analysis, phase equilibria, molecular spectroscopy, biosensors.

\section{INTRODUCTION}

Perhaps no other field has been the subject of such an intensive research interest nor received such intense attention as ionic liquids (ILs) are experiencing today. At its beginning, the field developed at a reasonable rate. In the 20 years elapsed from 1962 to 1981 there were about 65 publications in the topic, giving an average of 3.23 publications per year. In the next 20 years, from 1982 to 2001, this number increased to an average of 26.5 publications per year. However, 384 of the 530 papers published in this period appeared in the years 2000 and 2001, showing an impressive average of 192 publications per year. If this number seems already high, from 2002 to 2006 there were 4300 publications recorded and from 2007 to 2009 , over 8800 publications which had the words 'ionic liquids' in their titles. For those that are not in the field of ILs, as it is the case of the authors of this short note, it is desirable to have a guide to a key reading that will give basic introduction to this area. The purpose of this note is to help newcomers in this respect.

The year 2009 was especially active for IL researchers. The Third Congress on ILs [1], which took part in Cairns, (Australia), was preceded by a number of Pre-Symposia workshops dedicated to discussion of well defined topics. These events were circumscribed to those working in the field of ILs. On the other hand, two most timely texts for the use of researchers in general were published in 2009. Both texts were prepared with contributions of many experts, coordinated by one editor. The text "Ionic liquids: Physicochemical Properties" [2], Elsevier, 2009, with Professor Suojang Zhang, Editor, contains a wealth of information which usefulness is difficult to exaggerate. The text "Ionic Liquids in Chemical Analysis" [3], CRC Press, 2009, with Professor Mihkel Koel, Editor, presents a cohesive review of the extensive literature in the general subject of ionic liquids and although it has an emphasis on the applications to chemical

\footnotetext{
*Address correspondence to this author at the Department of Chemical Engineering, McGill University, 3610 University Street, Montreal, Que., H3A 2B2, Canada; Tel: (514) 398-4274; Fax: (514) 398-6678;

E-mail: juan.vera@mcgill.ca
}

analysis, its coverage is much more general. For this reason, we have decided to present here this review. This CRC publication is part of the CRC Analytical Chemistry Series with Professor Charles H. Lochmuller as Series Editor. Notably, Professor Lochmuller, in his Foreword to the text, indicates that applications of ILs to specific areas of chemical analysis are just at their beginnings and that there is much work to be done to have these fascinating substances used regularly in analytical procedures. On the other hand, in his Introduction to the text, Professor, Koel clearly emphasizes that the text goes well beyond the area of chemical analysis and it covers topics of interest for researchers working in many other areas of Chemistry and Chemical Engineering. He states: "This book is an attempt to collect experience and knowledge about the use of ILs in different areas of analytical chemistry such as separation science, spectroscopy, and mass spectrometry that could lead others to new ideas and discoveries. In addition, there are chapters providing information of studies on determination of physicochemical properties, thermophysical properties and activity coefficients, phase equilibrium with other liquids, and discussion of modeling, which are essential to know beforehand also for wider applications in analytical chemistry." If in this paragraph one exchanges twice the word 'analytical' by the words 'chemical engineering and', one gets a much better idea of the general usefulness of the material presented in this book. In fact, the purpose of this note is to call the attention of researchers from areas other than analytical chemistry to a book that could pass otherwise unnoticed due to its rather restrictive title. Thus, we will emphasize the parts of the book that are of general interest to researchers and pass rather superficially over the sections that are of interest to those working specifically in the field of analytical chemistry. We assume that researchers in the area of analytical chemistry would be naturally attracted to read this book due to its title.

\section{A Review Emphasizing Areas of General Interest}

The material presented in the book, which is subject of this note, [3] was prepared by 28 of the most active researchers in the field of ILs residing in 9 different countries. Of the 12 contributors from the USA, 7 are from the Oak Ridge 
National laboratory in Tennessee, 3 are from Notre Dame University, Indiana, and 2 are from Ohio universities. From other countries, Tallin University of Estonia, Queens University of Northern Ireland and Moscow State University of Russia have 3 contributors each. Spain and Germany have 2 contributors each and France, Denmark and Poland each participates with one contributor.

The book starts by presenting useful information even before its Introduction section. Immediately after the list of contributors, and before the Introduction, it presents a table with chemical names, structural formulas and abbreviations of the names for commonly used ILs. For a neophyte this table is a blessing and even for a researcher in a field using ILs it is a 'memory-aid' of special value. This table is supplemented in chapters 3 and 4 .

In the opinion of the authors of the present review, chapters 1 and 2 of the book are masterpieces of general interest. They stand by themselves and could perfectly well be considered as a monograph on the subject. In Chapter 1, Professor Urszula Domanska, from Warsaw University of Technology, Poland, single handled, gives an impressive overview of ILs and their properties. In 60 pages, under the title 'General review of ionic liquids and their properties', she presents a critical review that ranges from the effect of the structure of ILs on their physical-chemical properties to ILs phase equilibria of all kinds (solid-liquid, liquid-liquid and vapor-liquid). She includes activity coefficients, heat capacities and heat of mixing. The information is supported by 215 references, many of them from the year 2007 and even a few from the year 2008. Chapter 2, written by three researchers from Queens' University, Northern Ireland, (T. G. A. Youngs, C. Hardacre and C. L. Mullan), presents information on the experimental and theoretical determination of ILs structure. As the authors state in their introduction: "An understanding of the microscopic structure of liquids is paramount in the understanding of the properties of the system". They point out that this information is of assistance for narrowing the selection of potentially useful ILs for a particular application from tens of thousands to, perhaps, less than a hundred. They support their statements with 49 references, many of them from the year 2007 including their own unpublished results at the date of publication of the book.

The presentation of applications of ILs starts in Chapter 3. This chapter, written by five researchers from the Oak Ridge National Laboratory, Tennessee (S.N Baker, T.A. McCarty, F.V. Bright, W.T. Heller and G.A. Baker), gives an updated view, supported by 63 references, of the application of ILs to optical, electrochemical and biochemical sensor technology. Special emphasis is given to the use of ILs in biosensors. The chapter includes a table listing chemical names, structures and abbreviated names of ILs, which supplements the table with similar information for commonly used ILs presented at the beginning of the book, in front of the Introduction. As the authors state, the application of ILs to sensor technology is rather recent and it "is gaining extraordinary momentum." This is clearly seen from the list of references to this chapter in which, with only one exception, all references are from the last decade, many of them from 2007 and even one from 2008.
Chapters 4, 5, 6 and 7 are all related, in one way or another, to the use of ILs in chromatography. In Chapter 4, Professor J. L. Anderson from the University of Toledo, Ohio, discusses the use of ILs as stationary phases in gas chromatography. This chapter also includes a table with names, structures and physicochemical properties of selected ILs that have been evaluated as gas-liquid stationary phases. Chapter 5, written by Professor Apryll M. Stalcup from the University of Cincinnati, Ohio, presents the advances of the use of ILs in liquid chromatography; and Chapter 6, written by M. Vaher and M. Kaljurand from Tallin University of Technology, Estonia, discuss the use of ILs in capillary electrophoresis. Finally, in Chapter 7 an international group formed by two researchers from Spain (M.J. Ruiz-Angel and S. Carda-Broch) and one French researcher (A. Berthod) reviews the use of ILs as stationary phases in countercurrent chromatography.

Chapters 8, 9 and 10 deal with studies in phase equilibria and are a natural continuation of chapters 1 and 2 for the researchers outside the field of analytical chemistry. In Chapter 8 the group of the Department of Chemical and Biomolecular Engineering of the University of Notre Dame, Indiana, formed by J. F. Brennecke, Z. K. Lopez-Castillo and B.R. Mellein presents an authoritative overview of the solubilities of gases in ILs and the experimental techniques used for these measurements. The chapter includes a very useful table of Henry's Law constants for $\mathrm{CO}_{2}, \mathrm{CO}, \mathrm{O}_{2}, \mathrm{H}_{2}$ and $\mathrm{SO}_{2}$ in a variety of ILs. Chapter 9 , written by I.V. Pletnev, S. V. Smirnova and V. M. Egorov, from the Lomonosov Moscow State University, Russia, reviews the application of ILs for liquid-liquid extraction of organic compounds from aqueous mixtures and applications of ILs for treatment of nonaqueous solutions. After illustrating extraction processes with actual experimental data, these authors conclude saying that ILs are 'true designer solvents' and predict that their use will increase still further in the future as 'more finely tuned solvents' will be required. Closing this set of three chapters related to phase equilibria, Chapter 10 written by H. Luo and S. Dai from the Oak Ridge National Laboratory, Tennessee, presents the state of the art review, up to the year 2006, on the use of ILs for the separation of metal ions.

Chapters 11, 12, 13 and 14 are of interest to a researcher working in the field of spectroscopy. In the introductory Chapter 11, the editor of the book, Prof. Mihkel Koel, gives an excellent overview of current and possible future applications of ILs in different types of molecular spectroscopy due to their special properties, which can be often custom designed. Many researchers will find particularly interesting Section 11.4 discussing the various aspects of purity of ionic liquids. Chapter 12, written by Prof. Rolf W. Berg from the Technical University of Denmark in Lyngby, is devoted to theoretical and practical aspects of Raman spectroscopy used in studies of conformational equilibria in ILs. The author presents a detailed case study of the use of ab intio calculations together with Raman spectroscopy in structure determination of imidazolium-based ionic liquids. The local structures in ionic liquids and the temperature effect are also covered. A total of 109 references are presented, including the author's unpublished results. In Chapter 13, Prof. Ralf Giernoth from the University of Cologne (Germany) demonstrates that "Very much in contrast to common belief, the use of ILs as solvents does not preclude the application of NMR 
techniques." The numerous examples presented in the chapter show that virtually all standard and advanced NMR techniques can be used after careful adjustment of parameters. 74 references up to the year 2008 complement this excellent review. Chapter 14 is the last one dedicated to the spectroscopy area. Prof. Andreas Tholey from University of Saarland (Germany) focuses on two aspects of mass spectrometry (MS); its current and future application in almost all fields of IL research and the use of IL in MS itself, particularly as a matrix material in MALDI MS. The author observes that fundamental theoretical work has still to be done to better understand the relationship between the composition of an IL and its ability to serve as a good matrix.

\section{Additional Comments}

According to the Editor of the volume commented in this review [3], its writing had to be stopped in the middle of 2007. Although some of the chapters include references dated in 2008, judging by the statistics we presented in the Introduction, about 7000 titles have been published after the closing date. Thus, to keep track of what is presently going on on the rapidly growing field of ILs literature, one needs to consult more recent reviews. Among others, recent reviews by Pinto et al. [4]; Buszewski and Studzinska [5]; Zhu et al. [6], and K.A. Venkkatesan et al. [7] are of special interest. Considering the content of the book under review here [3], and the review of the use of ILs in chromatographic and electromigration techniques presented by Buszewski and Studzinska [5] it is important to repeat that the applicability of ILs goes well beyond the field of analytical chemistry [3, 5]. Pinto et al. [4] reviewed their use as reaction media for biocatalysis while Zhu et al. [6] comment on their use as green solvents. Similarly, Venkatesan et al. [7] discussed the use of ILs for reprocessing nuclear fuels and more recently it has been announced [8] that in the laboratory of Professor
Alexis Bell at Berkeley, CA, there are advanced studies for 'turning sunlight into fuel' by depolymerization of lignocellulose into fermentable sugars using ILs.

As a closing comment, it is necessary not to lose perspective over the potential hazards that can be created by the indiscriminate use of ILs. Zhu et al. [6] have discussed this point with complete clarity by saying that although the low vapor pressure of ILs may suggest that they are green solvents, these compounds can be released to the environment in many other possible ways such as accidental spills or effluents. In these cases, according to Zhu et al. [6], the ILs can "cause water and soil pollution, which depends on their bioaccumulation, toxicity and degradability." Certainly, these are points that are important to keep in mind.

\section{REFERENCES}

[1] http://www.coil-3.org/

[2] S. Zhang, Ed., Ionic Liquids: Physicochemical Properties, Elsevier: Amsterdam, USA, 2009.

[3] M. Koel, Ed., Ionic Liquids in Chemical Analysis, CRC Press: USA, 2009.

[4] P. C. A. G. Pinto, M. L. M. F. S. Saraiva and J. L. F. C. Lima, "Oxydoreductase behavior in ionic liquids in nuclear fuels: a review", Analytical Sciences, vol. 24, pp. 1231-1238, 2008.

[5] B. Buszewski and S. Studzinska, "A review of ionic liquids in chromatographic and electromigration techniques", Chromatographia, vol. 68, pp. 1-10, 2008.

[6] S. Zhu, R. Chen, Y. Wu, Q. Chen, X. Zhang and Z. Yu, "A minireview on greenness of ionic liquids", Chemical Biochemical Engineering, vol. 23, no. 2, pp. 207-211, 2009

[7] K. A. Venkatesan, T. G. Srinivasan and P. R. Rao, "A review on the electrochemical applications of room temperature ionic liquids in nuclear fuel cycle", Journal of Nuclear \& Radiochemical Science, vol. 10 (1), pp. R1-R6, 2009.

[8] Posted by Icyarris, "Turning Sunlight into Fuel: Ionic Liquid Diet Key to Unlocking Biomass Sugars", Berkeley Lab News Center, Available from: http://newsletter.lbl.gov/, April 2009. 